\title{
Labyrinthe
}

$27 \mid 2007$ (2)

La fin des disciplines?

\section{Défauts de savoirs}

\section{Laurent Dubreuil}

\section{(2) OpenEdition}

\section{Journals}

\section{Édition électronique}

URL : https://journals.openedition.org/labyrinthe/1892

DOI : 10.4000/labyrinthe.1892

ISSN : 1950-6031

\section{Éditeur}

Hermann

\section{Édition imprimée}

Date de publication : 4 juillet 2007

Pagination : 13-26

ISBN : 978-2-9526131-4-9

\section{Référence électronique}

Laurent Dubreuil, « Défauts de savoirs », Labyrinthe [En ligne], 27 | 2007 (2), mis en ligne le 25 mars 2011, consulté le 27 avril 2022. URL : http://journals.openedition.org/labyrinthe/1892 ; DOI : https:// doi.org/10.4000/labyrinthe.1892

Ce document a été généré automatiquement le 27 avril 2022.

Propriété intellectuelle 


\title{
Défauts de savoirs
}

\author{
Laurent Dubreuil
}

1 D'interdisciplinarité, nous parlons, et beaucoup. Nous en parlons entre nous qui faisons cette revue, entre chercheurs en général, de pays, de traditions, de positions différentes. Buzzword, disent les Américains : il bourdonne, il fait du bruit, et ne tilte peut-être pas. Nous nous passons le mot et débattons, avec tous les schématismes ordinaires des pros et des contras, sans oublier les attentistes. Mais ce qu'interdisciplinaire veut dire, vraiment, qu'en savons-nous? Le problème de la signification dans la langue, encore une fois. Le présent article sert d'introduction aux sens historiquement dominants d'interdisciplinaire, et, par là même, à ce numéro de Labyrinthe. Il n'est pourtant pas question pour moi de définir la catégorie, soit d'exclure des usages réputés indus, puis de montrer mon concept en disant voilà. Les plus belles définitions rationnelles sont au mieux capables d'établir une sous-catégorie (exemple, la raison selon Kant), qu'un accord de façade seulement autorise (exemples, les querelles entre kantiens). Cela ne conduit pas forcément à relativiser toutes choses. Plutôt, face à tout débat de mots - et de pensée -, nous pouvons qualifier et renoncer à définir, construire et nous passer d'édicter. Je préfère sans doute certains emplois d'interdisciplinarité, ceux qui escortent une audace intellectuelle m'attirent plus qu'un creux devenir technocratique. Il n'en reste pas moins que l'on ne règle pas la contradiction de la signification en élisant un sens triomphant. Les tentatives langagières les plus prescriptives, tel le dictionnaire, n'arrivent pas à tuer complètement la langue, tant mieux. Ne jouons pas à cela, ici. Donnons-nous les mots qui permettent d'indiquer nos propres limites, nos propres seuils, et à quels savoirs nous voudrions tenir. De temps à autre, pas toujours j'en conviens, quand même souvent, regardons quelles sont les relations de notre travail à la pensée, et nos manières, nos disciplines. Revenir encore sur l'interdisciplinaire n'est pas rester dans l'aridité supposée du méta-épistémique. En forçant un peu, comme j'aime à faire : c'est au contraire l'occasion possible d'un geste vital, rappelant à la réflexion locale combien elle doit des comptes à l'effort qu'est la pensée. Dit de la sorte, quelle ambition, certes; mais il nous la faut, si nous tentons de faire entendre par l'interdisciplinaire autre chose que l'effet d'institution. 


\section{Des mots, des sens}

2 Donc, nous partageons des mots, à défaut de signification. Le lexique est très loin d'être tout, pourtant quand un néologisme apparait a lieu une opération réflexive, qui nous soucie. D'où je commence par l'enquête lexicale, dans les textes français et anglais du siècle dernier. Si je laisse de côté les années 1990, trois moments se détachent dans l'usage collectif de l'interdisciplinaire au sein des sciences humaines. Il semble d'abord que le mot nouveau surgisse un peu avant 1930 et se répande dans les années immédiatement ultérieures. Ces premières occurrences se trouvent en rapport avec «l'École de Chicago » et l'organisation américaine nommée « Conseil pour la recherche en sciences sociales » (Social Science Research Council ou SSRC, fondé en 19231). Ce Conseil a valorisé, disséminé le terme, dans ses publications et, plus spécialement, par un système de prix et bourses alloués aux recherches "de nature interdisciplinaire ${ }^{2}$ ». Dans les années 1930, la jeune épithète interdisciplinaire est ainsi liée au contexte des sciences sociales d'abord et avant tout, à la sociologie, à la psychologie behaviouriste ${ }^{3}$. Elle désigne un type de recherche dont la nouveauté tiendrait à l'objet même. Ainsi se comprend l'énigmatique nature que je citais depuis l'appel à contributions du SSRC. La société, substrat des actions humaines, se comprend dans la confrontation des savoirs particuliers. Dans ce numéro, Anthony Mangeon revient sur la figure d'Alain Locke, dont la contribution à la science sociale de Chicago est généralement niée ou sousévaluée. Locke, face à la question noire dans la société américaine, se livrait à une réadaptation et transformation des limites disciplinaires par retour. Un peu plus tard (en 1946), Melville Herskovits comparera la "nature interdisciplinaire ${ }^{4}$ " des études afro-américaines à la composante « inter-continentale » de la traite des Noirs.

3 L'argument d'une nature conditionnant l'interdisciplinarité demeure vivace. Aux ÉtatsUnis, les area studies comme les études centrées sur des «minorités » (femmes, gays et lesbiennes, subalternes, postcoloniaux, etc.) justifient le plus souvent leurs structures inter-départementales par la condition «naturelle» de leur propre objet ${ }^{5}$. Au passage, l'argument s'adapte bien au constructivisme. On peut contester l'existence conceptuelle d'une nature - et garder une semblable démarche de légitimation. C'est ainsi que Homi Bhabha trouve dans l'hybridation culturelle un fondement théorique à l'interdisciplinarité des études postcoloniales ${ }^{6}$. Je crains hélas que la justification de l'interdisciplinaire ne soit douteuse chaque fois qu'elle présente la recherche comme l'image ou la réplique de ce qu'elle est censée saisir.

4 Après l'affirmation de la sociologie renouvelée, la deuxième période de promotion pour l'interdisciplinaire commence dans le second après-guerre. La pratique des area studies, qui se consolident à ce moment-là aux États-Unis, articule des connaissances discrètes à propos de zones géographiques. L'examen de ces régions concourait aux obsessions géopolitiques américaines ${ }^{7}$. Et là de nouveau, l'action du Comité pour la recherche en sciences sociales est notable. En parallèle, l'Unesco (créée en 1946) joue un rôle crucial : elle assure la publicité de l'interdisciplinaire comme idéal savant. Menés sous l'égide de l'Organisation, colloques et rapports recommandent les rencontres entre disciplines (cross-disciplinary revient en sus d'interdisciplinary). Une telle façon de travailler se présente désormais comme la meilleure manière de faire de la recherche en général - et non plus seulement au sujet de la société. La logique de correspondance entre science et objet se maintient, mais l'enjeu est plus large. De même que les nations se doivent d'être unies, de minimiser leurs différences et d'ouvrir leurs frontières, la 
recherche d'après-guerre a pour tâche d'abandonner au plus vite les vieilles divisions, de transgresser les limites des spécialités et départements universitaires. Les actes de la première "conférence internationale de la recherche pédagogique " (édités en 1956), conseillent d'aller " au plus vite au-delà des frontières nationales individuelles ${ }^{9}$ ", de s'instruire sur "la recherche [menée] dans d'autres pays, et d'autres disciplines ${ }^{10}$ »; bref de bâtir un enseignement "interdisciplinaire ${ }^{11}$ ». Quelques années plus tard, un rapport américain, sponsorisé par l'Unesco critique les «cloisonnements intellectuels artificiels " qui régissent "les disciplines universitaires» au profit d'un dialogue théorique rejoignant «les préoccupations des Nations unies et d'autres organisations internationales ${ }^{12} »$. L'interdisciplinarité serait donc requise par une situation internationale. Dans cette phase particulière de mondialisation, l'interconnexion entre les sciences passe pour la mission historique d'une époque. Le constat est d'autant plus pressant pour le "Tiers monde» dont les structures éducatives sont à reconstruire après l'accession à l'indépendance. Il n'est pas accidentel que la première revue, apparemment, à se nommer Interdiscipline émane d'un institut sis à Varanasi, en Inde ${ }^{13}$. L'avertissement " aux lecteurs », au début du premier numéro d'Interdiscipline, combine les différentes justifications épistémiques. L'auteur, Sugata Dasgputa, y affirme qu'il faut à «la recherche en sciences sociales » une pratique «interdisciplinaire ${ }^{14}$ ». Plus encore, "le terme "inter-disciplinaire" évoque un concept entièrement nouveau ", celui d'" une nouvelle région d'études ${ }^{15} »:$ a new area of study qui, dans le langage du savant postcolonial, inverse le syntagme des impérialistes area studies. Cette région prétendue nouvelle a en fait un double site, le monde et l'Inde. Le monde puisque le règne de la "fusion inter-disciplinaire " est annoncé, qui nous donnera une "vue holistique ${ }^{16}$ " du réel. L'Inde dans la mesure où «la valeur d'une approche interdisciplinaire devient plus évidente $a$ fortiori dans un pays comme le nôtre, dont la tâche principale est d'enclencher un processus rapide de développement social ${ }^{17} \%$. Par conséquent, les «disciplines en voie de développement ${ }^{18}$ » résultent d'une alliance entre les secteurs traditionnels du discours savant et les propices étais de la science dans un PVD.

5 Le troisième temps fort de l'interdisciplinaire paraît plus éloigné de cet appel au consensus mondial si typique de l'Unesco. Nous entrons dans une ère de rébellions et de révolutions. Particulièrement, la France, dans la suite de Mai 68, devient le lieu de publication d'un abondant corpus où l'interdisciplinarité est, au choix, prescrite ou proscrite. Le mot interdisciplinaire existe en français bien avant 1968, mais, pour l'essentiel, se restreint à des essais technocratiques en langue Unesco, ou aux ouvrages de chercheurs en sciences sociales qui connaissent l'Université américaine ${ }^{19}$. Dans les années qui précèdent Mai, il est utilisé par des professeurs qui développent une critique acide de l'enseignement supérieur français et trouvent précisément dans l'adjectif un moyen de désigner ce que ce système n'est pas. L'un de ces contestataires de l'intérieur est Georges Gusdorf ${ }^{20}$. Ce dernier, pour son credo interdisciplinaire, est cité en épigraphe d'un tract distribué en 1965 par des enseignants de philosophie à la Faculté de Nanterre (où les "événements" débuteront). Le même tract formule trois exigences: " 1 . Pédagogie de la synthèse et de l'universalité./ 2. Ensemble de la civilisation contemporaine. / 3. Recherche interdisciplinaire ${ }^{21}$. "

6 Après Mai 68, la connivence entre l'interdisciplinarité et le refus de la vieille Sorbonne du savoir est si nette que des observateurs sont conduits à croire qu'interdisciplinaire a été inventé par la jeunesse estudiantine ${ }^{22}$. L'impression est inexacte au plan lexicographique. Elle rend toutefois l'évidence : interdisciplinarité s'impose en français 
grâce à Mai, puis aux expérimentations pédagogiques du Centre universitaire de Vincennes. On peut douter malgré tout de l'importance de la revendication interdisciplinaire durant l'occupation de la Sorbonne. J'aurais envie de penser que l'interdisciplinarité, grâce aux événements de Mai, quitte le rang marginal de vœu pieux parmi quelques universitaires critiques et se mue en impératif théorique pour des intellectuels dont l'audience s'élargit à compter de 1968. Michel de Certeau, JeanMarc Coudray ou Guy Michaud - pour nous en tenir à trois chercheurs qui, séparément, publient en 1968 des essais favorables à Mai - annoncent tous une réforme du savoir qui ruinera les antiques divisions dans la connaissance ${ }^{23}$.

7 Ici et là, la séparation est prise pour une barrière, une frontière, une limite. L'interdisciplinarité est une brèche, qui va décloisonner ${ }^{24}$. Parmi les amateurs d'interdisciplinarité, l'intérêt pour la spatialisation du savoir, pour l'architecture de la pensée, est plus que métaphorique. L'École de Chicago se constitue par l'étude des répartitions géographiques et sociales dans la ville, elle se conjoint à l'urbanisme ${ }^{25}$. L'Université en questions de Gusdorf s'ouvre sur une comparaison entre les bâtiments ordinaires des universités français et les vastes campus d'Angleterre ou d'Amérique. Le rapprochement fonctionne comme illumination: "C'est le néant architectural de l'Université française qui m'a fait comprendre son néant intellectuel et spirituel ${ }^{26}$. À la fin de Révolution dans l'Université, Guy Michaud, qui souhaite "reconstruire l'Université ${ }^{27}$ ", demande un "contact réel avec la citée ${ }^{8}$ " rompant avec le ban de la banlieue nanterroise et l'implantation de «campus isolés du monde par des talus de chemin de fer et de routes express ${ }^{29}$ ». « Pour l'Université que nous voulons », ajoute-til, «le choix du site est essentiel ${ }^{30} »$. Dans tous ces cas, l'interdisciplinarité semble le remède à l'isolement. Elle désigne un changement possible pour le site de la pensée (universitaire, au moins), à la croisée des chemins, à l'intersection des connaissances. Elle se présente comme le puissant moyen de modifier la configuration de l'espace intellectuel, ouvrant des brèches dans la forteresse, jetant des ponts entre les rives. Et, en même temps, d'altérer l'endroit même où recherche et enseignement ont lieu, l'enseignement supérieur, les universités, etc. La chose se comprend selon ces deux dimensions. Le cas du séminaire, que Marc Aymes analyse dans ces pages, est un lieu spectral de la recherche, de l'éducation, du campus. Le site est l'un des termes pour la zone complexe, ce lieu étrange, qui crée et que créent les savoirs.

\section{Le renouveau de l'ancien}

8 La célébration de l'interdisciplinaire coïncide-t-elle magiquement avec la création d'une recherche renouvelée ? Voire. L'interdisciplinarité sert régulièrement à rajeunir la dénomination de vieilles habitudes. Malgré les rumeurs contestataires, l'unitarisme méthodique peut s'accommoder assez bien de l'interdisciplinarité. Par un paradoxe efficient, on prépare alors la fusion de la pluralité dans le monisme interdisciplinaire, qui se croit ensemble des ensembles, union mystique des savoirs séparés, fondement de toute réflexion ou culminance des connaissances. Il surprend un peu de lire, dans le premier numéro du Journal of Interdisciplinary History (en 1970), le slogan « Nous serons catholiques à la fois conceptuellement et géographiquement ${ }^{31}$.» La terre d'une telle interdisciplinarité conceptuelle est le paradis ou le domaine du Grand Tout, divinement révélé par une recherche enfin universelle. Ajoutons qu'une autre revue, la seule, je crois, à se baptiser Journal of Interdisciplinary Studies entend mener une décisive " percée 
dans la compréhension de Dieu, de l'homme et de l'univers ${ }^{32}$ ». Je ne dis pas que l'interdisciplinarité est structurellement vouée à chercher la localisation précise du Royaume de Dieu. Je ne veux pas même suggérer que le croisement des savoirs accomplisse toujours un retour sous d'autres formes de la raison théologique (et chrétienne en particulier). Mais assurément le lexique que nous observons s'adapte à merveille au jargon de l'unité de la sagesse.

Question subsidiaire : qui pourrait accomplir semblable synthèse, et où? Mettons de côté - peut-être trop vite, au fond - la figure du chercheur illuminé dont la force intérieure (venue du travail, du génie, de la science, de Dieu) causerait le miracle de l'interdisciplinarité. Il nous reste alors un ancestral débat sur ce que les scolastiques nommaient disciplina disciplinarum. En d'autres termes, ou presque les mêmes, une discipline serait plus apte que les autres à donner un sens à l'interdisciplinarité. Deux opiniâtres prétendantes au trône sont l'histoire et la philosophie. Il se pourrait aussi que l'unification théocratique de la connaissance eût lieu sur un autre plan, supérieur à toutes disciplines, celui d'un paradigme unifiant. Des options aussi divergentes que la Gestalttheorie, le cognitivisme ou la théorie (plus ou moins) française sont de récents candidats à l'investiture. À chacune de ces occasions, l'exigence d'interdisciplinarité unit le disjoint selon un principe de pouvoir. Le motif théologique est donc le symptôme d'une persistance de l'ordinaire dans le domaine du savoir. Dans ce cadre, toute répartition « interdisciplinaire » finit par mettre au goût du jour les controverses sur les hiérarchies célestes. Au mieux, des configurations antérieures périront au profit d'autres; mais la forme de l'espace même (où les positions sont possibles) demeure intouchée.

10 Se méfiant du grand Pan, ou le croyant mort, une interdisciplinarité moyenne et plus neutralisée préfèrera des fusions locales à la participation holistique. Inter renvoie à interaction ici et légitime la collaboration entre chercheurs issus de différents champs. Ce sens restreint de l'entreprise, j'en ai l'impression, est la position de repli la plus acceptable pour les logiques d'institutions. Ce que l'université américaine regroupe sous le label de "programmes » est le lieu officiel d'un travail collectif, collaboratif et focused. Entre tant d'autres, il en va ainsi du programme French Studies à Cornell, cet endroit virtuel qui abrita, dans l'enceinte matérielle du campus, une part de la réflexion aboutissant au présent numéro. French Studies est financé, reconnu, administré comme le locus d'interactions limitées au sujet polymorphe qui le rend possible ${ }^{33}$. Dans les sciences dures, dont nous avons choisi de peu parler cette fois, le modèle collaboratif d'interdisciplinarité détient la primauté. L'intégration partielle de l'informatique et de la médecine, par exemple, donne de nouveaux agrégats - qui ne questionnent pas les disciplines. Parmi les sciences humaines, les studies américaines, elles, sont autant d'interzones pour des rencontres étranges. Elles forment au maximum des sections jusque-là inédites dans le corps du savoir, qui pourraient affaiblir les disciplines traditionnelles - ce qui, entre autres, explique l'horror sacer de la majorité des universitaires français à l'encontre de ces reconfigurations. Il va de soi, toutefois, que ces inter tendent à contingenter de nouveau, avec pas moins de forces que les disciplines. Les questions du type de "comment relier les Africana Studies avec les French Studies et les Gay and Lesbian Studies? " ne font que déplacer les interrogations du genre de « quels liens créer entre l'anthropologie, la philosophie et l'histoire?».

11 Ces réflexions en matière d'interdisciplinarité auraient tôt fait de moi une espèce de croyant sceptique. Ce pourrait être vrai, pas totalement pourtant. Je souligne 
simplement que la foi dans l'interdisciplinarité réside en bien des lieux. Sauf exception, et malgré des effets répandus de grandiloquence, ce qui se nomme interdisciplinaire ne détruit ni ne révolutionne l'architecture de la pensée. David Schreiber et Renaud Pasquier, dans leur texte dialogique, indiquent plus tard les précipices où l'on s'abîme souvent, les fossés entre effets et faits des interdisciplines. Faut-il s'étonner? Après tout, l'interdisciplinaire (qu'il soit unitaire, hégémonique, collaboratif, paradigmatique ou local) dépend toujours de la légende du polymathe. Par légende j'entends une chose chimérique, et, autant, vitale, liée plus ou moins secrètement à toute diction. Légende également, car cela se dit, se répète, les mots différant avec une identique syntaxe. Je choisis polymathie pour cette envie d'étudier, utiliser, comprendre plus d'une science. Le mousikos anèr, l'Humaniste de la Renaissance, l'Encyclopédiste, le généraliste sont des polymathes, ce qui ne signifie pas qu'ils sont parfaitement pareils. Au contraire, chaque situation, époque, histoire aurait construit l'image d'un chercheur et penseur maximaliste. L'interdisciplinarité ne serait qu'une présentation contemporaine. Je soupçonne nombre d'institutions aujourd'hui « interdisciplinaires » d'avoir été naguère des écoles "généralistes ». Juste avant la vogue lexicale lancée par ses sociologues, l'influence de l'Université de Chicago fut déterminante lors de la bataille pédagogique autour du généralisme dans le premier cycle universitaire aux États-Unis ${ }^{34}$. En France, l'École normale supérieure, que les auto-célébrations du bicentenaire peignirent comme le locus amoenus de l'interdisciplinarité35, était en 1836, d'après Victor Cousin, le seul établissement où la " culture générale » s'articulât à chaque "spécialité »"

L'interdisciplinarité renvoie au site du savoir où l'infini se crée de la confrontation avec la multiplicité du fini. Elle est susceptible d'apporter un contrepoids nécessaire au succès actuel de l'expertise locale de légitimation (dont abusent la technocratie politique, le pouvoir managérial à l'affût de consultants, les philosophies " communicationnelles » et scolastiques-analytiques). Ce n'est pas rien. Elle pourrait déboucher, aussi, sur la simple variante d'un invariant ethos du chercheur, désigner une attitude aujourd'hui contestée mais nettement plus ancestrale que son propre nom.

\section{Sur un concept trouble}

13 Les hypostases que je viens d'indiquer ne devraient pas nous inciter à réfuter en bloc l'interdisciplinarité sous prétexte des contradictions qui s'y logent. Oui, interdisciplinaire souvent recèle un concept trouble. Seulement, tant de mots font de même. Nous pouvons résister à la tentation de fixer le bon usage. On peut se récrier contre la confusion entre inter- et pluri-disciplinaire - et je le fis plus d'une fois. Cette bizarre appropriation a une histoire que Laurent Ferri explique dans ce numéro. Le choix du pluri en français marque le signe d'un compromis politique. Edgar Faure, ministre de l'Éducation nationale, donna l'impression d'accéder à la demande soixante-huitarde d'interdisciplinarité en inscrivant le pluri dans la loi, mais en laissant ouvert ce que ce nouveau préfixe signifierait (choisissez un ou plusieurs : juxtaposition administrative, continuation du modèle français d'enseignement généraliste, interactions théoriques, insurrection du concept). D'un autre côté, le pluri (ou le multi, comme préfèrent dire les locuteurs anglais) était déjà contenu dans l'interdisciplinaire. Ce mot n'a jamais eu de fixité que dans la critique de la séparation (entre contingentements du savoir, entre l'université et la société, etc.). Le déferlement néologique qu'interdisciplinary a provoqué (avec, en franglais, les adjectifs pluri-, multi-, cross-, trans-, in-, post- et même mluri- 
disciplinair ${ }^{37}$ ) révèle la séduction exercée par la critique et l'impossible consensus sur le sens, tout autant que la quête de l'illimité par la limitation. L'interdisciplinaire, malgré ses métamorphiques et secrètes connivences avec la positivité totalitaire, est ce qu'on en fera. Il nous revient de construire ses significations par l'écriture, la parole, la lecture, le travail. Nous ne mettrons pas fin à la controverse, parce que l'existence du débat sémantique a du sens. Les conceptualisations les plus pédagogiques restent sans conséquence réelle lorsqu'elles veulent ignorer la défectivité de l'interdisciplinaire à travers ses occurrences insistantes. Qu'interdisciplinarité ne veuille pas dire une seule chose est un défaut - mais à partir duquel nous devons penser. Le seul trait commun à tous les «interdisciplinaristes » tient à ce qu'inter est au minimum interruption (de l'interruption), beaucoup plus qu'intersection.

14 Passant par chaque discipline avec un sérieux jusqu'au-boutiste, nous venons aux points de ligature. La couture se défait. Autrement dit, chaque science doit se poursuivre au-delà d'elle-même, comme ce qu'elle n'est pas (ou ne saurait être). Non pas compléter l'une par l'autre. Rendre sensible l'inaptitude de chacune par sa propre mise en œuvre puis sa trahison dans l'autre savoir ; ainsi de suite. Est-ce trop demander aux mots de l'interdisciplinarité ? Je ne suis pas sûr, bien qu'avec d'autres dans cette revue, j'ai tenté l'indiscipline afin de faire résonner une négativité, une pratique autre. La paronomase est un effet supplémentaire de la construction de la signification. Elle n'est pas inouïe; en 1971, Charles Debbasch écrivait déjà « rien n'est plus indiscipliné que le pluridisciplinaire ${ }^{38}$ ». Jacques Rancière, après que nous l'avions qualifié d'indiscipliné dans un numéro spécial de Labyrinthe paru en 2004, a récemment promu l'indisciplinaire ${ }^{39}$. Je préfère en rester à indiscipline et indiscipliné, faisant de ces termes des rebelles au suffixe didactique (en -aire) et les qualificatifs d'un ethos du chercheur. Si interdisciplinaire signifie ce que je dis, si dans ce texte cela s'entend, j'ai la contrainte liberté de mon propre langage, idiosyncrasique et à tous vents ouverts.

Que l'interdisciplinarité recouvre tant de contradictions sémantiques, qu'elle désigne les négativités du savoir n'est pas sans rappeler la situation de la littérature dans le dispositif des connaissances. De ce point de vue indiscipliné, la littérature exerce une influence sans commune mesure avec sa place dans la "culture». Ici, l'enjeu du littéraire est l'intégration d'autres discours dans son corpus, un réarrangement qui n'est ni parachèvement ni imitation. La capacité poétique fissure le concept par l'exhibition de la signification dans la langue, opération dont elle se retrouve une sorte de garante ${ }^{40}$. Richard Klein explore dans ce dossier les failles interdisciplinaires et la négativité littéraire. Je poserais le problème autrement, en n'identifiant rien de crucial pour la littérature dans la fiction. Pour moi, la signification littéraire défait la trame conceptuelle du langage que des millénaires de disciplines intellectuelles ont tenté de former. Mais elle brode ensuite, ne nous laissant pas dans le seul instant du négatif. Enfin, ce découpage, ce tissage n'existent qu'après l'avènement de connaissances instituées. La mise en œuvre littéraire des paroles donne les moyens de comprendre le défaut de la pensée disciplinaire. Ce que la critique philosophique apporta naguère aux sciences humaines, une reprise de la littérature peut le déplacer encore. Ce qui nécessite que les interprètes des œuvres se mettent à l'école des autres savoirs - et surtout qu'ils abjurent l'intenable croyance dans une quelconque supériorité des études littéraires sur d'autres disciplines. Ce que la littérature exprime n'est pas identique à ce qu'elle nous permet, et il faut inventer encore. Que ce soit clair, en son sens institutionnel, la critique littéraire n'a pas plus de vertus naturelles que d'autres 
disciplines. Elle a plutôt la malheureuse habitude de détruire toute espèce de signification vivante par une pratique typologique, et n'est donc pas « bien placée " par principe. Que le commerce avec la littérature entretienne la pensée explique pourquoi le littéraire a tant compté pour des historiens, des philosophes, des anthropologues, des linguistes des dernières décennies. Les départements de littérature sont juste censés s'occuper un peu mieux que les autres du poétique; l'expérience laisse deviner que l'évidence est tout sauf automatique. Il s'agit donc bien de l'indiscipline que met en œuvre la littérature : celle-ci peut nous aider à proposer un autre mode de connaître, non réductible à l'enseignement des lettres, l'interprétation ou l'herméneutique. Ambitieuse, voire prétentieuse visée, que nous avons à garder en nous pourtant, si nous cherchons à énoncer quoi que ce soit de signifiant. Inventer, donc, à partir de ce qui est. Ce texte, en son lieu, quel est-il ? Je ne sais rien de certain, mais si j'osais répondre, je dirais peut-être une critique littéraire de l'épistémologie, ou la poétique d'un mot dans l'histoire philosophique.

L'interdisciplinaire n'a pas de sens, si l'on veut dire ceci, cela, un point c'est tout. Ce mot, dont on se passa si bien avant le siècle dernier, pourrait de lui-même tomber dans l'avenir. L'urgence qu'il lui arrive de signifier, pourtant, ne l'a pas attendu et devrait le dépasser. Les impasses disciplinaires ne sont jamais que des figures de notre impossible. L'interdisciplinaire que motive le manque peut nommer la démarche de persévérance dans l'effondrement - mieux que la croquignolesque assomption du plein. Voyons cependant ce que l'indiscipline accorde ; elle n'est pas une fin. Elle ne prend pas sens à défaut, mais au défaut du savoir que la pensée produit.

Avec quels langages on renouvelle la connaissance; selon quels modes nous nous cherchons des espaces pour jouer aux savants; par où nous cessons de pouvoir penser et pourquoi ; comment tirer profit du savoir institué sans rester dans l'illusoire autofondation; si la séparation épistémique est nécessaire, contingente, obsolète, adventice, négligeable ; voilà quelques-uns des problèmes que l'interdisciplinarité nous ferait reprendre. Pour répondre, il faudrait plusieurs vies. Raison de plus pour continuer. Créons des marges, des corps étrangers, des villes intérieures. À nous d'autres sites, d'autres architectures. Faisons une revue. Tiens, c'est déjà fait; changeons encore les choses, alors. Intervenons sur nos interlocutions, replaçons, déplaçons. Rédigeons un dossier entier sur l'interdisciplinaire, pour ne plus y revenir, pour être certains d'y retourner. Soyons ensemble et séparés, juste nous, ce mouvant nous, plus que nous (et moins aussi, vous le deviniez). Interrompez-nous si vous ne voulez pas. Interdisciplinaires? indisciplinons!

\section{NOTES}

1. Lire Kenton Worcester, Social Science Research Council, 1923-1998, p.32 sqq. Le rapport est disponible en ligne, à l'adresse http://www.ssrc.org/publications/about_the_ssrc/ SSRC_History.pdf [consulté le 15 mai 2007].

2. Citation provenant de l'annonce des prix signée en 1933-1934 par le «Dr. Donald Young, Secrétaire pour les bourses et allocations décernées par la Conseil pour la recherche en sciences 
sociales ». Ma source est plus particulièrement ici Social Forces, vol. XI, n 4, 1932, p. 588, mais l'appel se trouve dans d'autres revues. La même expression (" de nature interdisciplinaire ») se retrouve dans les appels des années 1934-35 et 1935-36. - Ici et plus bas, les traductions de l'anglais sont les miennes.

3. Voir par exemple Walter Hunter, "Research Interests in Psychology », American Journal of Psychology, vol. LIV, n 4, p. 605.

4. Melville J. Herskovits, « Problem, Method and Theory in Afroamerican Studies », Phylon, 1946, p. 337-354 (publié plus tôt dans Afroamerica, vol. I, n 1-2, 1945). Herskovits va plus loin encore, rapprochant le cross-disciplinary (croisement disciplinaire) du race crossing (croisement de races), dernier sujet auquel il avait consacré The American Negro. A Study in Racial Crossing, New York, Knopf, 1928 : voir dans l'article cité les p. 338, 340, 341, 342.

5. Les lecteurs peu familiers de ce genre de structures universitaires américaines peuvent se reporter à l'entretien avec Anne Berger, «Traversées de frontières : postcolonialité et études de "genre" en Amérique », Labyrinthe. Atelier interdisciplinaire, n² 24, 2006, p. 11-37.

6. Je renvoie à mon article "Alter, inter : académisme et postcolonial studies ", Labyrinthe, $\mathrm{n}^{\circ} 24$, 2006, p. 47-62. Le livre de Homi Bhabha, The Location of Culture, Londres/New York, Routledge, 1994, vient d'être traduit en français sous le titre Les Lieux de la culture. Une théorie postcoloniale, Paris, Payot, 2007.

7. Voir Immanuel Wallerstein, «The Unintended Consequences of the Cold War on Area Studies ", dans Noam Chomsky (éd.)., The Cold War and the University, New York, New York Press, 1997.

8. On peut se reporter au programme emblématique de Wendell C. Bennett, Area Studies in American Universities, New York, Social Science Research Council, 1951.

9. Report of the First International Conference on Educational Research, Paris, Unesco, 1956, p. 7. En accord avec les statuts de l'Unesco, il existe une version française publiée sous le titre Conférence internationale de la recherche pédagogique. Depuis mon Amérique, je n'ai pu consulter la traduction autorisée et me reporte donc ici au texte anglais.

10. Report..., op. cit., p. 12.

11. Idem, p. 29.

12. Toutes citations venant de American Education in a Revolutionary World: The Role of the States, Albany, The University of the State of New York, 1964, p.23. L'ouvrage mentionne la «Commission américaine auprès de l'Unesco » parmi ses sponsors.

13. Interdiscipline: A Quaterly Journal Devoted to Social Science Research and Documentation, vol. III, $\mathrm{n}^{\circ} 1$, [Gandhian Institute of Studies, Varanasi, Inde] 1966. Ce titre prend la suite de Social Science Abstracts.

14. Idem, p. I.

15. Ibidem.

16. Ibidem pour les deux citations de cette phrase. L'auteur utilise la graphie wholistic, qui insiste encore davantage sur la totalité (whole). L'adjectif se retrouve p. IV.

17. Idem, p. I.

18. Idem, p. III. Je pousse ici la traduction de «developing disciplines» (developing country est l'équivalent de pays en voie de développement).

19. Jean Meynaud, professeur à l'Université de Lausanne, visait à fonder une science politique francophone sur la " coopération interdisciplinaire ", entre autres choses (voir son Introduction à la science politique, Paris, Colin, 1959, p. 258). Parmi les références récurrentes de l'ouvrage figurent les rapports rédigés sous l'égide de l'Unesco et des ouvrages américains récents de sciences sociales (voir idem, p. 7-11 et 260 sqq.).

20. Georges Gusdorf, L'Université en question, Paris, Payot, 1964.

21. Texte reproduit dans Guy Michaud, Révolution dans l'université, Paris, Hachette, 1968, p. 141. 
22. Par exemple Robert Flacelière, Normale en péril, Paris, Presses universitaires de France, 1971, p. 59.

23. Voir Michel de Certeau, La Prise de parole. Pour une nouvelle culture, Paris, Desclée de Brouwer, 1968, p. 114-115 ; Edgar Morin, Claude Lefort, Jean-Marc Coudray, Mai 1968 : la brèche. Premières réflexions sur les événements, Paris, Fayard, 1968, p. 140 ; Guy Michaud, Révolution dans l'université, p. 9 et 98.

24. Voir le titre même de Morin et al., Mai 1968 : la brèche, et, au sein de cet ouvrage, les attaques contre les «cloisonnements» (p. 140). La métaphore correspondante en anglais est la décompartementalisation.

25. Voir, en français, l'anthologie dirigée par Yves Grafmeyer et Isaac Joseph, L'École de Chicago. Naissance de l'écologie urbaine, Paris, Fayard, 2004.

26. Gusdorf, L'Université en question, op. cit., p. 8-9.

27. Michaud, Révolution dans l'université, op. cit., p. 139.

28. Idem, p. 137.

29. Ibidem.

30. Ibidem. Rappelons au passage que Henri Lefebvre, qui travaillait sur l'urbanisme des villes nouvelles et des banlieues, était l'un des professeurs de Nanterre.

31. The Journal of Interdisciplinary History, vol. I, $\mathrm{n}^{\circ} 1,1970$, p. 4.

32. Journal of Interdisciplinary Studies: An International Journal of Interdisciplinary and Interfaith Dialogue, vol. I, $\mathrm{n}^{\circ} 1 / 2,1989$, deuxième de couverture.

33. Sujet polymorphe car, même dans l'esprit de son directeur, l'élément French déborde de l'area vers la pensée écrite en français, des éléments de civilisation, ainsi que les mondes francophones. 34. Sur la question de l'éducation dite générale dans le premier cycle universitaire américain, lire Frederick Rudolph, Curriculum. A History of the American Undergraduate Course of Study Since 1636, San Francisco, Jossey-Bass, 1977, p. 236 sqq.

35. Voir L'Apprentissage du savoir vivant. Fonction des grands colleges européens, Paris, Presses universitaires de France, 1995, livre édité pour le «Bicentenaire de la fondation de l'École normale supérieure » : p. 93-96 (texte de Jacques Le Goff), p. 144-145 («transgress[er] les limites [des] discipline[s] », chez Christophe Charle); et, sous le label de "pluridisciplinarité », p. 150 (article collectif d'élèves de l'École) et p. 183 (Éric Fassin à propos de la «pluridisciplinarité normalienne »).

36. Victor Cousin, « Rapport sur les travaux de l'École normale pendant l'année 1835-1836 », dans École normale. Règlements, programmes, et rapports, Paris, Hachette, 1837, p. 187 pour les deux éléments cités.

37. Pluri- et multi- existent tous deux en français et anglais. Cross-disciplinary est très répandu en anglais, dès les années 1950. Transdisciplinarité est surtout employé par des auteurs tels qu'Edgar Morin et Basarab Nicolescu ; lire, du dernier, Transdisciplinarité. Un Manifeste, Monaco, Le Rocher, 1996 (traduit aux États-Unis en 2002); voir également le site électronique du «Centre de recherches et études transdisciplinaires " à l'adresse http://nicol.club.fr/ciret/index.htm [consulté le 15 mai 2007]. Outre ce que je dis plus loin sur indisciplinaire chez Jacques Rancière, je renvoie à Laurent Loty "Pour l'indisciplinarité » (in Studies on Voltaire and the Eighteenth Century, $2005, n^{\circ} 4$, p. 245-259), p. 245, note 1, pour une mise au point lexicographique, et à l'ensemble de l'article pour sa propre position. En anglais, les jeux de mots sur undisciplined et indiscipline sont moins directs, et, pour l'instant, moins présents (voir néanmoins, à part Rancière donc, l'article de 2005 de Rosemarie Scullion «Reaching Out: Interdisciplinarity, Foreign Language Departments, and the Liberal Arts Curriculum », réédité dans Profession, [s. 1.], The Modern Language Association of America, 2006, p. 130). Postdisciplinarity caractérise, selon Louis Menand, la situation présente de la scholarship (The Marketplace of Ideas, New York, American Council of Learned Societies, 2001, p. 13-14 par exemple). Enfin, mluridisciplinarité est l'intéressant lapsus calami que Laurent Ferri exploite dans la suite de ce numéro. 
38. Charles Debbasch, L'Université désorientée. Autopsie d'une mutation, Paris, Presses universitaires de France, 1971, p. 70.

39. Titre du dossier : "Jacques Rancière l'indiscipliné » (Labyrinthe, $\left.\mathrm{n}^{\circ} 17,2004\right)$; je redis que l'adjectif apparaît depuis 2003 dans l'argumentaire de notre revue. Lire Jacques Rancière, "Thinking between the disciplines : an aesthetics of knowledge », traduit en anglais par Jon Roffe (depuis un texte inédit en français, à ma faillible connaissance), Parrhesia, I, 1, 2006, p. 9-12. Voir encore l'autoprésentation des actes du Colloque de Cerisy sur Rancière, qui parle en outre d'indisciples : La Philosophie déplacée. Autour de Jacques Rancière, Paris, Horlieu, 2006.

40. Je fais allusion ici à des analyses que je mène ailleurs, et avec plus de détail, sur la pensée littéraire, la signification langagière et la ruine du concept. Pour aller au plus court, lire «Des raisons de la littérature ", Labyrinthe, $\mathrm{n}^{\circ} 14,2003$, p. 11-24, et, tout récemment, "What Is Literature's Now? », New Literary History, 2007, n 1, p. 43-70.

\section{AUTEUR}

\section{LAURENT DUBREUIL}

Ld79(at)cornell.edu 